\title{
Diet and Prey Selection of Barn Swallows (Hirundo rustica) at Vancouver International Airport
}

\author{
Audrey A. Law ${ }^{1,6}$, Miranda E. Threlfall ${ }^{1,2}$, Brendon A. Tijman ${ }^{1,3}$, Eric M. Anderson ${ }^{1}$, Sean \\ MCCANN $^{4}$, GARY SEARING ${ }^{5}$, and DAVID BRADBEER ${ }^{5}$

\begin{abstract}
${ }^{1}$ British Columbia Institute of Technology, 3700 Willingdon Avenue, Burnaby, British Columbia V5G 3H2 Canada
${ }^{2}$ Current address: 5651 Chester Street, Vancouver, British Columbia V5W 3B3 Canada

${ }^{3}$ Current address: 8851 Ash Street, Richmond, British Columbia V6Y 3B4 Canada

${ }^{4}$ Simon Fraser University, 8888 University Drive, Burnaby, British Columbia V5A 1S6 Canada

${ }^{5}$ Vancouver International Airport, 3211 Grant McConachie Way, Richmond, British Columbia V7B 0A4 Canada

${ }^{6}$ Corresponding author: audreyalaw@hotmail.com
\end{abstract}

Law, Audrey A., Miranda E. Threlfall, Brendon A. Tijman, Eric M. Anderson, Sean McCann, Gary Searing, and David Bradbeer. 2017. Diet and prey selection of Barn Swallows (Hirundo rustica) at Vancouver International Airport. Canadian FieldNaturalist 131(1): 26-31. https://doi.org/10.22621/cfn.v131i1.1777

The Barn Swallow (Hirundo rustica) is the most widely distributed aerial insectivore in North America, but has declined appreciably in recent decades. Reasons for these declines are largely unknown, though presumably relate mainly to changes in prey availability. To help inform conservation priorities for this species, we assessed their diet and prey selection using birds lethally struck by aircraft at Vancouver International Airport (YVR). Esophagi and gizzards of 31 Barn Swallows collected from June 2013 to October 2013 contained insects mainly from the orders Hymenoptera (mean across birds $=40 \%$ of insect numbers), Diptera (31\%), Hemiptera (15\%), and Coleoptera (12\%). To assess prey selection, we compared the esophagi and gizzard contents of 20 swallows collected from July 2013 to September 2013 to populations of aerial insects we sampled during the same period using Malaise traps. Barn Swallows selected strongly for insects in the order Hymenoptera (mainly Formicidae, which comprised $29 \%$ of diet), and selected against insects in the orders Coleoptera, Diptera, and Lepidoptera. For all prey taxa combined, Barn Swallows displayed strong selection for insects of length 4-8 mm (body length excluding appendages). Conversely, they selected against smaller and larger insects, despite the fact that smaller insects comprised about $80 \%$ of all insects sampled in Malaise traps. Combined with past studies, our results suggest that Barn Swallows select among available aerial insects within local feeding sites for taxa that (i) are of intermediate size, (ii) occur at relatively high density, and (iii) have poor flight performance.

Key Words: Barn Swallow; Hirundo rustica; aerial insectivore; diet; prey selection; Vancouver International Airport; British Columbia

\section{Introduction}

Populations of aerial insectivores in Canada have declined by $70 \%$ since 1970 , constituting the most pronounced decline among all categories of Canadian birds (North American Bird Conservation Initiative Canada 2012). The Barn Swallow (Hirundo rustica) is the most widely distributed aerial insectivore in North America and has declined appreciably throughout Canada, with Breeding Bird Survey data for 1970-2011 showing a mean annual trend of $-4.01 \%$ (Environment Canada 2013). Barn Swallows were assessed as Threatened in 2011 by the Committee on the Status of Endangered Wildlife in Canada (COSEWIC), but have yet to be listed under the Species at Risk Act (COSEWIC 2011; British Columbia Conservation Data Centre 2013; Government of Canada 2017). The causes of Barn Swallow declines are unknown, but likely include a combination of the following threats on breeding grounds: loss of nesting and foraging sites due to agricultural changes, decreased abundance of aerial insects, climate change, competition for nest sites with House Sparrows (Passer domesticus), ectoparasites, and nest removal by humans (COSEWIC 2011).
Very few detailed diet studies have been completed for Barn Swallows, particularly for the North American subspecies (H. r. erythrogaster) that differs in behaviour and morphology from the Eurasian subspecies $(H . r$. rustica; Brown and Brown 1999). Limited results for both subspecies suggest that prey size is an important factor affecting prey selection; although small prey items occurred in the diet, Barn Swallows fed mainly on relatively large prey ( $\geq 1.5 \mathrm{mg}$ dry mass; Turner 1982; Holroyd 1983). Adult Barn Swallows also tend to feed larger prey to their young than they eat themselves (Waugh 1978). A sample of 467 Barn Swallows collected throughout the United States and Canada contained mainly Diptera (39.5\%), Hymenoptera (22.7\%), Coleoptera (15.6\%), and Hemiptera (15.1\%; method of analysis not specified; Beal 1918).

The purpose of this study was to assess the diet and prey selection of an at-risk aerial insectivore, the Barn Swallow. To study this sensitive species, we took advantage of birds lethally struck by aircraft at Vancouver International Airport (YVR) on Sea Island in Richmond, British Columbia, Canada $\left(49.2^{\circ} \mathrm{N}, 123.2^{\circ} \mathrm{W}\right)$. Barn Swallows are a concern at YVR because since 
2000 they have accounted for about $35 \%$ of identified birds struck by aircraft annually (D.B., unpublished data). To assess prey selection, we compared the gut contents of Barn Swallows to populations of aerial insects we sampled throughout YVR airfields. These results may help reduce the frequency of Barn Swallow strikes at YVR if the abundance of key prey items can be managed.

\section{Methods}

\section{Barn Swallow carcass collection and dissection}

We obtained Barn Swallows $(n=31)$ from the YVR airfield after they had been lethally struck by aircraft between 2 June 2013 and 1 October 2013 (specific collection dates were unknown for two swallows obtained during this period). Swallows were not randomly sampled and therefore are not necessarily representative of all Barn Swallows at YVR. Carcasses were collected from the runway $<10$ min after they were struck, and then stored at $-20^{\circ} \mathrm{C}$ until they were dissected. For dissections, birds were opened ventrally and the gastrointestinal tract was removed. An incision was made down the entire length of the esophagus and the gizzard and all their contents were flushed and preserved in $95 \%$ ethanol until they were processed. The contents of intestines were discarded because digestion rendered them unidentifiable.

\section{Availability of insect prey}

We collected samples of insect relative abundance on the YVR airfield from five Malaise traps $(156 \mathrm{~cm}$ width $\times 170 \mathrm{~cm}$ height; Figure 1). Malaise traps pro-

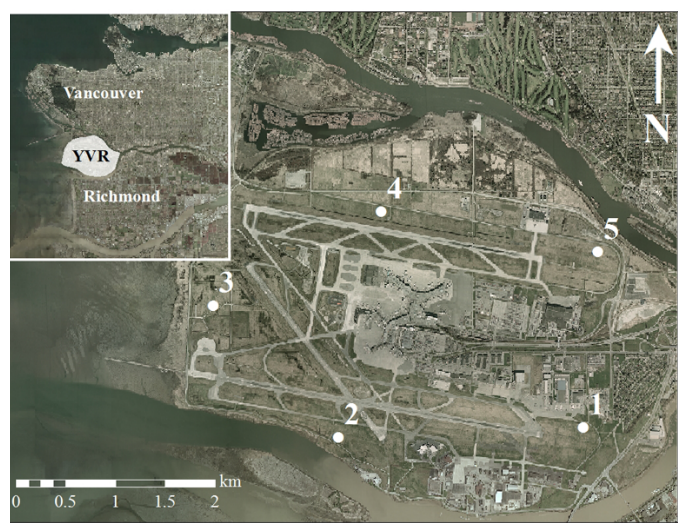

FIGURE 1. Locations of sampling sites for the insect prey of Barn Swallows (Hirundo rustica) at Vancouver International Airport (YVR). At each of the five sites, Malaise traps were left open from 4 July 2013 to 12 September 2013 during which weekly samples were obtained of insect prey availability ( $n=50$ total samples). Photo: Esri, Redlands, California, USA, 2009.

vide a reliable method of capturing a diverse taxonomic range of flying insects. An exception is Coleoptera that are only captured in Malaise traps at a rate $25 \%$ of that using rotary, glass-barrier, or sticky traps (Juillet 1963; Pickering 1994). Malaise traps were distributed evenly throughout the airfield in areas that would not interfere with airport operations. Malaise traps were left open from 4 July 2013 to 12 September 2013, and samples were gathered weekly from each trap $(n=50$ total samples). Samples were stored in jars of $95 \%$ ethanol at room temperature until they were processed.

\section{Insect identification}

Insect specimens from the Malaise traps and from Barn Swallow esophagi and gizzards were identified using dissecting microscopes (8-32×) and taxonomic keys (Triplehorn and Johnson 2005). Specimens in Barn Swallow guts were identified to family, and then further sorted into three size classes: small $(0-4 \mathrm{~mm}$ body length excluding appendages), medium (4-8 mm), and large $(>8 \mathrm{~mm})$. In two cases we sorted insects only to the level of infraorder (Pentatomomorpha, Culicomorpha) and in one case to the level of superfamily (Muscoidea) because many individuals in these groups could not reliably be identified to family. Barn Swallow consumption of many insect families observed in Malaise traps was too low to reliably assess swallow prey selection at the level of family. Thus, prey selection and identification of Malaise trap specimens were conducted only at the level of order. All non-aerial insects, such as spiders, were excluded from Malaise trap samples because Barn Swallows are aerial insectivores. Only one spider was found in the 31 Barn Swallow esophagi and gizzards.

\section{Analysis of Barn Swallow prey selection}

We calculated selection ratios separately for insect orders and size classes. However, to avoid biased estimates we did not calculate selection ratios for several orders that were rare (i.e., mean values of $<1 \%$ ) in either Barn Swallow esophagi and gizzards or Malaise traps. Selection ratios were calculated by dividing the mean percentage of insects that Barn Swallows had consumed by the mean percentage sampled in the Malaise traps (Manly et al. 2002). Selection ratios $>1$ indicate selection for a prey category, while selection ratios $<1$ indicate selection against a prey category. Separately for selection ratios based on insect orders and size classes, we also calculated $95 \%$ simultaneous confidence intervals using the Bonferroni inequality as follows:

$$
\mathrm{SR} \pm\left[\mathrm{z}_{\alpha /(2 I)} \times \mathrm{SE}(\mathrm{SR})\right]
$$

where $\mathrm{SR}$ is a selection ratio, $\mathrm{SE}$ is its standard error, $\mathrm{z}_{\alpha}$ is the value that is exceeded with probability $\alpha=0.05$ by a standard normal random variable, and $I$ is the number of selection ratios considered (Manly et al. 2002). Collection dates for nine of the 31 Barn Swallows occurred either 32 days before or 6-19 days after the period of Malaise trapping, and exact collection dates for two Barn Swallows were unknown. Thus, for prey selection analysis, we used only the 20 Barn Swallows that were gathered during Malaise trap sampling; col- 
lection dates of these Barn Swallows were evenly distributed throughout the period of Malaise trap sampling. Moreover, the relative composition of orders did not vary markedly during the period of Malaise trap sampling: percentages of Diptera and Hemiptera declined slightly, and percentages of Hymenoptera, Lepidoptera, and Psocoptera increased slightly (Figure S1).

\section{Results}

Barn Swallow diet

We identified 514 individual insects representing eight orders in the esophagi and gizzards of the 31 Barn Swallows (Table 1). Barn Swallows consumed insects mainly from the orders Hymenoptera (40\%), Diptera (31\%), Hemiptera (15\%), and Coleoptera (12\%). The Hymenoptera included mainly Formicidae (ants), the
Diptera included mainly Calliphoridae (blow flies), and the Hemiptera included mainly Pentatomomorpha (seed bugs and stink bugs). The most prevalent of the ants recovered from Barn Swallows were males and queens of Myrmica specioides and an unidentified species of Myrmica. Of the Hemiptera consumed, most (mean across birds $=78 \%$ of insect numbers) belonged to the suborder Heteroptera (true bugs), with a large proportion of these representing a single species of Coreidae, Ceraleptus pacificus.

\section{Barn Swallow prey selection}

A total of 8608 individual insects representing 12 orders were collected from Malaise traps. We calculated selection ratios for five orders that comprised $\geq$ $1 \%$ of mean values for both Barn Swallow esophagi

TABLE 1. Mean percentages of insect numbers (with 95\% CIs) and percent frequency of occurrence (\%FO) of insect orders and families identified in esophagi and gizzards of Barn Swallows (Hirundo rustica; $n=31$ ) collected at YVR after they had been lethally struck by aircraft during 2 June 2013 to 1 October 2013. Results include two infraorders (Culicomorpha, Pentatomomorpha) and one superfamily (Muscoidea) within which many individuals could not reliably be identified to family.

\begin{tabular}{|c|c|c|c|c|c|c|c|c|}
\hline & \multicolumn{2}{|c|}{ All Sizes } & \multicolumn{2}{|c|}{ Small $(<4 \mathrm{~mm})$} & \multicolumn{2}{|c|}{ Medium $(4-8 \mathrm{~mm})$} & \multicolumn{2}{|c|}{ Large $(>8 \mathrm{~mm})$} \\
\hline & $\begin{array}{l}\text { Mean \% } \\
(95 \% \text { CI })\end{array}$ & $\% \mathrm{FO}$ & $\begin{array}{l}\text { Mean \% } \\
(95 \% \text { CI })\end{array}$ & $\% \mathrm{FO}$ & $\begin{array}{c}\text { Mean \% } \\
(95 \% \text { CI })\end{array}$ & $\% \mathrm{FO}$ & $\begin{array}{l}\text { Mean \% } \\
(95 \% \text { CI })\end{array}$ & $\% \mathrm{FO}$ \\
\hline Araneae & $0.3(0.6)$ & 3.2 & $0.3(0.6)$ & 3.2 & - & - & - & - \\
\hline Coleoptera & $12.2(6.7)$ & 45.2 & $8.1(4.5)$ & 38.7 & $4.1(4.4)$ & 19.4 & $0.1(0.2)$ & 3.2 \\
\hline Cantharidae & $0.2(0.3)$ & 3.2 & - & - & $0.2(0.3)$ & 3.2 & - & - \\
\hline Chrysomelidae & $1.1(1.4)$ & 9.7 & $1.0(1.4)$ & 6.5 & $0.1(0.2)$ & 3.2 & - & - \\
\hline Curculionidae & $0.6(0.9)$ & 6.5 & $0.6(0.9)$ & 6.5 & - & - & - & - \\
\hline Elateridae & $0.5(1.1)$ & 3.2 & - & - & $0.5(1.1)$ & 3.2 & - & - \\
\hline Staphylinidae & $1.9(3.4)$ & 6.5 & - & - & $1.8(3.4)$ & 3.2 & $0.1(0.2)$ & 3.2 \\
\hline unidentified spp. & $7.9(5.6)$ & 32.3 & $6.5(4.3)$ & 29.0 & $1.5(2.8)$ & 6.5 & - & - \\
\hline Diptera & $30.5(13.6)$ & 67.7 & $11.4(7.6)$ & 48.4 & $15.9(11.7)$ & 35.5 & $3.2(6.3)$ & 3.2 \\
\hline Calliphoridae & $11.6(10.6)$ & 22.6 & $1.2(2.3)$ & 6.5 & $7.2(8.8)$ & 12.9 & $3.2(6.3)$ & 3.2 \\
\hline Chloropidae & $0.1(0.1)$ & 3.2 & $0.1(0.1)$ & 3.2 & - & - & - & - \\
\hline Culicomorpha & $5.4(6.0)$ & 25.8 & $5.4(6.0)$ & 25.8 & - & - & - & - \\
\hline Dolichopodidae & $4.3(3.8)$ & 29.0 & $4.3(3.8)$ & 29.0 & - & - & - & - \\
\hline Muscoidea & $5.1(6.1)$ & 12.9 & - & - & $5.1(6.1)$ & 12.9 & - & 一 \\
\hline Psyllidae & $0.03(0.1)$ & 3.2 & - & - & $0.03(0.1)$ & 3.2 & - & - \\
\hline Sciomyzidae & $3.2(6.3)$ & 3.2 & - & - & $3.2(6.3)$ & 3.2 & - & - \\
\hline Sphaeroceridae & $0.2(0.4)$ & 3.2 & - & - & $0.2(0.4)$ & 3.2 & - & - \\
\hline Tipulidae & $0.2(0.3)$ & 6.5 & - & - & $0.2(0.3)$ & 6.5 & - & - \\
\hline unidentified spp. & $0.4(0.8)$ & 3.2 & $0.4(0.8)$ & 3.2 & - & - & - & - \\
\hline Hemiptera & $15.2(7.4)$ & 51.6 & $5.9(5.7)$ & 19.4 & $9.4(5.9)$ & 35.5 & - & - \\
\hline Aphididae & $3.2(3.8)$ & 12.9 & $3.2(3.8)$ & 12.9 & - & - & - & - \\
\hline Corixidae & $0.1(0.3)$ & 3.2 & - & - & $0.1(0.3)$ & 3.2 & - & - \\
\hline Miridae & $1.3(1.7)$ & 9.7 & $0.4(0.7)$ & 3.2 & $1.0(1.6)$ & 6.5 & - & - \\
\hline Pentatomomorpha & $10.5(7.0)$ & 29.0 & $2.3(4.4)$ & 3.2 & $8.3(5.9)$ & 25.8 & - & - \\
\hline Hymenoptera & $40.0(13.0)$ & 77.4 & $6.8(5.3)$ & 41.9 & $33.0(12.8)$ & 61.3 & $0.2(0.3)$ & 3.2 \\
\hline Braconidae & $0.3(0.4)$ & 6.5 & $0.3(0.4)$ & 6.5 & - & - & - & - \\
\hline Chalcidoidea & $0.1(0.2)$ & 3.2 & - & - & $0.1(0.2)$ & 3.2 & - & - \\
\hline Chrysididae & $1.0(1.4)$ & 6.5 & $0.4(0.7)$ & 3.2 & $0.6(1.3)$ & 3.2 & - & - \\
\hline Formicidae & $29.2(12.3)$ & 51.6 & - & - & $29.2(12.3)$ & 51.6 & - & - \\
\hline Halictidae & $1.4(1.3)$ & 12.9 & $0.6(0.8)$ & 6.5 & $0.7(1.0)$ & 6.5 & - & - \\
\hline Pteromalidae & $1.1(2.1)$ & 3.2 & $1.1(2.1)$ & 3.2 & - & - & - & - \\
\hline Tenthredinidae & $0.3(0.5)$ & 6.5 & $0.1(0.3)$ & 3.2 & $0.2(0.4)$ & 3.2 & - & - \\
\hline unidentified spp. & $6.6(4.2)$ & 51.6 & $4.4(3.8)$ & 35.5 & $2.1(2.4)$ & 12.9 & $0.2(0.3)$ & 3.2 \\
\hline Lepidoptera & 0.9 (1.4) & 6.5 & - & - & - & - & $0.9(1.4)$ & 6.5 \\
\hline Odonata & $0.5(0.8)$ & 6.5 & $0.1(0.3)$ & 3.2 & - & - & $0.4(0.7)$ & 3.2 \\
\hline Coenagrionidae & $0.1(0.3)$ & 3.2 & $0.1(0.3)$ & 3.2 & - & - & - & - \\
\hline unidentified spp. & $0.4(0.7)$ & 3.2 & - & - & - & - & $0.4(0.7)$ & 3.2 \\
\hline Thysanoptera & $0.4(0.7)$ & 3.2 & $0.4(0.7)$ & 3.2 & - & - & - & - \\
\hline
\end{tabular}




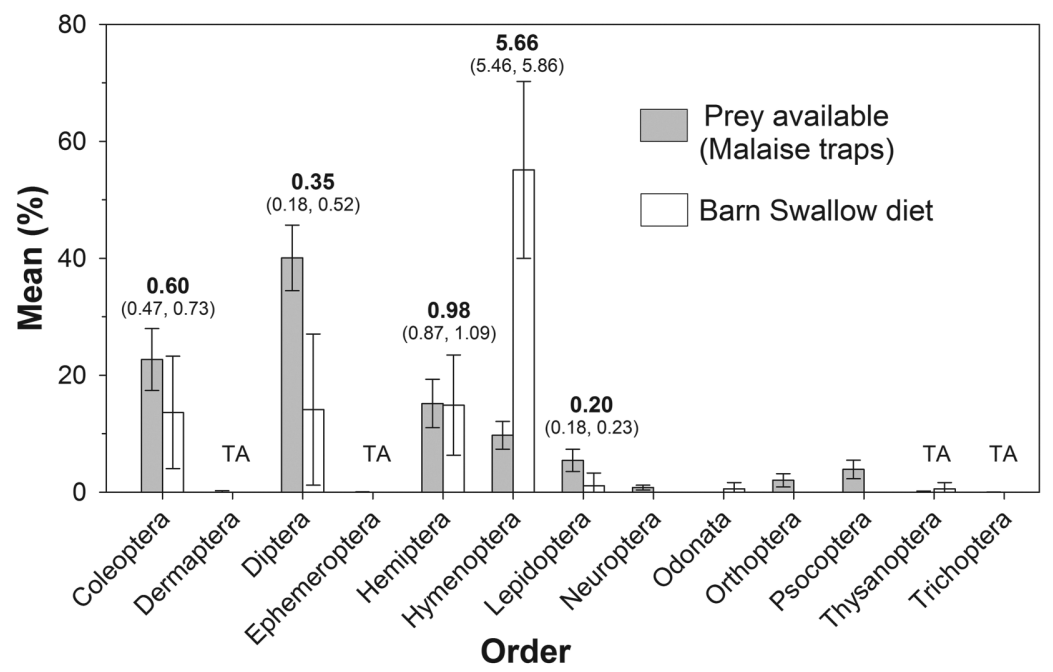

FIGURE 2. Mean percentages of insect numbers (with 95\% CIs) for orders (including all size classes) that were sampled in Malaise traps $(n=50)$ or in the esophagi and gizzards of Barn Swallows (Hirundo rustica; $n=20)$ that had been struck by aircraft at YVR. Selection ratios with 95\% Bonferroni simultaneous CIs are depicted above bars of insect orders for which mean values of prey available and prey consumed by Barn Swallows were both $\geq 1 \%$. Malaise traps included all orders except Odonata, but the following orders were not consumed by Barn Swallows: Dermaptera, Ephemeroptera, Neuroptera, Orthoptera, Psocoptera, and Trichoptera. TA (trace available) indicates insects that were present in Malaise trap samples but comprised $<1 \%$. Both available prey and prey consumed by Barn Swallows were sampled from 4 July 2013 to 12 September 2013.

and gizzards and Malaise traps (Figure 2). Of these, Barn Swallows selected for insects in the order Hymenoptera, selected against insects in the orders Coleoptera, Diptera, and Lepidoptera, and showed no preference for or against insects in the order Hemiptera (i.e., the $95 \%$ confidence interval for the Hemiptera selection factor included 1). For all prey taxa combined, Barn Swallows displayed selection for insects in the medium size class (4-8 mm; Figure 3 ). Conversely, they selected against large insects $(>8 \mathrm{~mm})$ and small insects $(0-4 \mathrm{~mm})$, despite the fact that small insects comprised about $80 \%$ of all insects sampled in Malaise traps.

\section{Discussion}

Barn Swallows consumed a wide range of insect taxa at YVR, but over half their diet consisted of just three taxa: Hymenoptera: Formicidae (29\%), Diptera: Calliphoridae (12\%), and Hemiptera: Pentatomomorpha (11\%). Our results are consistent with past studies in both North America and Europe indicating that during the breeding period both Barn Swallow adults and nestlings (via their parents) consume aerial insects mainly in the orders Coleoptera, Hymenoptera, Diptera, and Hemiptera (Beal 1918; Holroyd 1983; Kopij 2000; Turner 2006; Orłowski and Karg 2011, 2013). However, Barn Swallow diets differed appreciably among studies in terms of their relative consumption of insects in these four orders as well as their reliance on insects at finer taxonomic levels. Our results, together with those of past studies, suggest that Barn Swallows

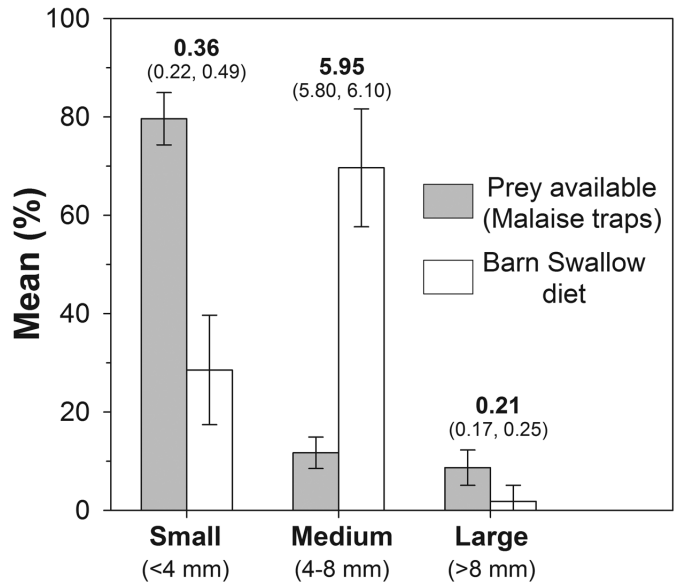

Size class

FIGURE 3. Mean percentages of insect numbers (with 95\% CIs) for small, medium, and large size classes (all taxa combined, body length excluding appendages) that were sampled in Malaise traps $(n=50)$ and in the esophagi and gizzards of Barn Swallows (Hirundo rustica; $n=$ 20) that had been struck by aircraft at YVR. Selection ratios with $95 \%$ Bonferroni simultaneous CIs are depicted above bars for each size class. Both available prey and prey consumed by Barn Swallows were sampled from 4 July 2013 to 12 September 2013.

do not rely on consistent prey taxa across sites, but instead select among available aerial insects within local feeding sites for taxa of intermediate size, weak flight 
performance (i.e., low speed and maneuverability), and that occur at relatively high densities. Formicidae, the most heavily consumed taxa in this study, appear to satisfy these three criteria.

Barn Swallows at YVR selected intermediate sizes of aerial insects (4-8 mm body length) that comprised just over $10 \%$ of available prey, while selecting against small insects $(<4 \mathrm{~mm})$ that comprised nearly $80 \%$ of available prey. Although differences in techniques used to measure insect size restrict direct comparison, past studies similarly indicated that intermediate to large insects are selected by adult Barn Swallows (Turner 1982; Holroyd 1983) and are fed to nestlings (Orłowski and Karg 2011, 2013). For instance, insects $>0.5 \mathrm{mg}$ dry body mass comprised just $14 \%$ of available insects, yet constituted $50 \%$ of nestling diets in rural Poland (Orłowski and Karg 2011). Compared to small insects, large insects yield greater net energy gains to Barn Swallows despite requiring greater foraging effort (Turner 2006). Thus, selection against Coleoptera and Diptera by YVR Barn Swallows was likely due in part to the mainly smaller sizes of these prey (74\% of Coleoptera and $95 \%$ of Diptera in Malaise traps were $<4 \mathrm{~mm}$ ). Further, we may have underestimated selection against Coleoptera because Malaise traps may under-sample insects in this order (i.e., Malaise traps may sample only about $25 \%$ of the numbers obtained using rotary, glass-barrier, or sticky traps; Juillet 1963). The profitability of insect prey may decline above a body size threshold given that Barn Swallows at YVR selected against the largest insects $(>8 \mathrm{~mm})$. Selection against Lepidoptera may have resulted, in part, from the mainly large sizes of insects in this order $(62 \%$ of Lepidoptera in Malaise traps were $>8 \mathrm{~mm}$ ). The relative benefits of larger prey may decline when smaller prey are highly abundant or are easily captured due to weak flight performance; these two factors appeared to underlie the significant consumption of small-bodied Formicidae and Ichneumonidae by Barn Swallows in several breeding sites in Poland (Orłowski and Karg 2011). The size of prey consumed by breeding Barn Swallows is also positively correlated with the distance between feeding and nesting sites (Waugh 1978; Turner 2006).

The profitability of prey to Barn Swallows also appears to increase with the tendency of prey to aggregate in dense swarms. For instance, the two most heavily consumed families of prey in our study included Formicidae and Calliphoridae, presumably because insects in these families commonly aggregate to mate (Formicidae) or to feed (Calliphoridae; Triplehorn and Johnson 2005). Past studies of Barn Swallow diets also reported significant consumption of Formicidae and Calliphoridae (Beal 1918; Orłowski and Karg 2011). The species most heavily consumed by YVR Barn Swallows was likely $M$. specioides (Formicidae), based on our detailed assessment of 2013 swallows as well as preliminary observation of swallows that were lethally struck in 2014 (S.M., unpublished data). Myrmica specioides is an invasive ant that was first observed in British Columbia in 2011 (R. Higgins, personal communication).

Declines in Barn Swallow populations appear to be due, in part, to reduced abundance of their aerial insect prey resulting from factors such as climate change, wetland loss, light pollution, and agricultural intensification (e.g., loss of pastureland, increased pesticide use; Evans et al. 2007; COSEWIC 2011). Formicidae appear to be a relatively unique prey type that increases in urban settings (McIntyre 2000), and managing the distribution or abundance of Myrmica ants at YVR may help reduce strikes of Barn Swallows by aircraft. Whether insect management programs at airports succeed in reducing strikes of aerial insectivores will require a thorough knowledge of the life history and ecology of these bird species as well as their insect prey.

\section{Acknowledgements}

The YVR Wildlife Management Program was instrumental in collecting Barn Swallow carcasses and insect samples, as well as graciously providing workspace and equipment. Paul Levesque collected the insect samples from the Malaise traps. Rob Higgins, Thompson Rivers University, provided expertise in ant identification on 1 February 2014. Lastly, we thank Tom Saare and the BCIT Renewable Resources Department for lending us additional lab equipment.

\section{Literature Cited}

Beal, F. 1918. Food habits of the swallows, a family of valuable native birds. Bulletin. United States Department of Agriculture, No. 619. https://doi.org/10.5962/bhl.title.108 596

British Columbia Conservation Data Centre. 2013. Species summary: Hirundo rustica. B.C. Ministry of Environment. Accessed 4 September 2015. http://a100.gov.bc.ca/pub /eswp/speciesSummary.do?id=17978.

Brown, C. R., and M. B. Brown. 1999. Barn Swallow (Hirundo rustica). The Birds of North America Online. Edited by A. Poole. Cornell Lab of Ornithology, Ithaca, New York, USA. Accessed 4 September 2015. https://birdsna.org/Spe cies-Account/bna/species/barswa.

COSEWIC (Committee on the Status of Endangered Wildlife in Canada). 2011. COSEWIC assessment and status report on the Barn Swallow Hirundo rustica in Canada. Ottawa. Accessed 4 September 2015. http://www.sararegis try.gc.ca/virtual_sara/files/cosewic/sr\% $\% 5$ Fbarn $\% 5$ Fswal low\%5F0911\%5Feng\%2Epdf.

Environment Canada. 2013. Trend results for Barn Swallow. North American Breeding Bird Survey - Canadian Trends Website, Data Version 2011. Environment Canada, Gatineau, Quebec. Accessed 4 September 2015. http:// www.ec.gc.ca/ron-bbs/P004/A001/?lang=e\&m=s\&r= BARS\&p=-

Evans, K. L., J. D. Wilson, and R. B. Bradbury. 2007. Effects of crop type and aerial invertebrate abundance on foraging barn swallows Hirundo rustica. Agriculture, Ecosystems \& Environment 122: 267-273. https://doi.org/10 .1016/j.agee.2007.01.015 
Government of Canada. 2017. Species at Risk Public Registry, Species Profile for Barn Swallow. Accessed 26 January 2017. http://sararegistry.gc.ca/species/speciesDetails e.cfm?sid=1147\#ot 18 .

Holroyd, G. L. 1983. Foraging strategies and food of a swallow guild. Ph.D. thesis, University of Toronto, Toronto, Ontario, Canada.

Juillet, J. A. 1963. A comparison of four types of traps used for capturing flying insects. Canadian Journal of Zoology 41: 219-223. https://doi.org/10.1139/z63-023

Kopij, G. 2000. Diet of swifts (Apodidae) and swallows (Hirundinidae) during the breeding season in South African grassland. Acta Ornithologica 35: 203-206. https://doi.org /10.3161/068.035.0201

Manly, B. F. J., L. L. McDonald, D. L. Thomas, T. L. McDonald, and W. P. Erickson. 2002. Resource Selection by Animals: Statistical Design and Analysis for field Studies. Second edition. Kluwer Academic Publishers, New York, USA. https://doi.org/10.1007/0-306-48151-0

McIntyre, N. E. 2000. Ecology of urban arthropods: a review and a call to action. Annals of the Entomological Society of America 93: 825-835. https://doi.org/10.1603/0013-87 46(2000)093[0825:EOUAAR]2.0.CO;2

North American Bird Conservation Initiative Canada. 2012. The State of Canada's Birds, 2012. Environment Canada, Ottawa, Canada. Accessed 4 September 2015. www .stateofcanadasbirds.org/State_of_Canada's_birds_2012.pdf.
Orlowski, G., and J. Karg. 2011. Diet of nestling Barn Swallows Hirundo rustica in rural areas of Poland. Central European Journal of Biology 6: 1023-1035. https://doi.org /10.2478/s11535-011-0070-4

Orlowski, G., and J. Karg. 2013. Partitioning the effects of livestock farming on the diet of an aerial insectivorous passerine, the Barn Swallow Hirundo rustica. Bird Study 60: 111-123. https://doi.org/10.1080/00063657.2012.748 717

Pickering, J. 1994. Calibration of Malaise traps for studying insect diversity. Discover Life. Accessed 4 September 2015. http://www.discoverlife.org/pa/or/idp/PROPOSALS/nsf. 94. final.html.

Triplehorn, C. A., and N. F. Johnson. 2005. Borror and DeLong's Introduction to the Study of Insects. Seventh edition. Thomson Learning, Inc., Brooks/Cole, Belmont, California, USA.

Turner, A. K. 1982. Optimal foraging by the swallow (Hirundo rustica, L): prey size selection. Animal Behaviour 30: 862-872. https://doi.org/10.1016/S0003-3472(82)80160-7

Turner, A. K. 2006. The Barn Swallow. T. \& A. D. Poyser, London, United Kingdom.

Waugh, D. R. 1978. Predation strategies in aerial-feeding birds. Ph.D. thesis, University of Stirling, Stirling, United Kingdom.

Received 23 March 2016

Accepted 26 January 2017

\section{SUPPlementaRy MATERIAL:}

FIGURE S1. Percentages of insects by order sampled from all five malaise traps during July ( $n=15$ total samples), August ( $n=25$ total samples), and September ( $n=10$ total samples) 2013. Orders with low abundance were grouped in the category 'Other' (orders Dermaptera, Ephemeroptera, Thysanoptera, and Trichoptera). 